\title{
Marked swelling with coalescing ecchymoses of the lower mandible in a Xenopus laevis frog
}

Coralie Zegre Cannon, $B A^{1}$, Keith Linder, DVM, PhD, DACVP ${ }^{1}$, Brenda J. Brizuela, $P h D^{2}$ \&

Stephen B. Harvey, DVM, MS, DACLAM ${ }^{2}$

A female African clawed frog, Xenopus laevis, was housed at the Biological Resources Facility of North Carolina State University in a tank with two other females. The frog was $\sim 1.5$ years old and was originally acquired from Xenopus Express (Plant City, FL) in 2003. The tank water was routinely changed three times a week using a fill-dump system. In this system, the water was not completely drained out in order to keep the frogs covered with approximately 1-2 inches of water during the tank water change. The frogs were fed a pelleted diet that consisted of Sinking-Frog-Food (3/32 inch diameter, Xenopus Express).

The animal care staff reported one frog with a swelling on the lower right mandible. No other animals in the colony were affected. On physical examination, the frog appeared alert and responsive. However, to ensure a definitive diagnosis, after consultation with the investigator, the animal was euthanized in buffered MS-222 so we could perform a complete necropsy.

Initial examination at necropsy revealed a marked swelling of the right mandible with coalescing ecchymoses on the left oral commissure (Fig. 1). Examination of the oral cavity revealed multiple $0.2-$ $0.3 \mathrm{~cm}$ mucosal ulcers on the dorsal hard palate, mandibular mucosa, and ventral oropharynx. Examination of coelemic organs revealed a grossly enlarged and

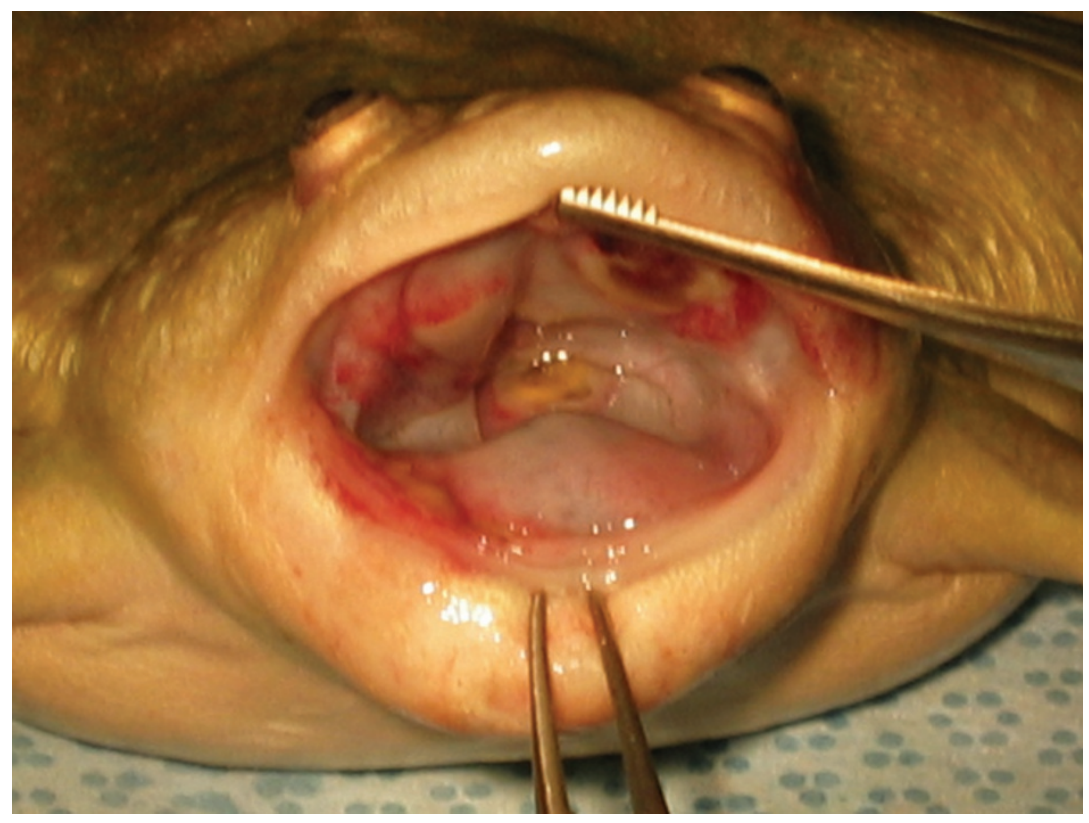

FIGURE 1 | The oral cavity of a 1.5-year-old, female African clawed frog (Xenopus laevis) at necropsy. The frog had exhibited a marked swelling of the right mandible, and at necropsy, ecchymotic hemorrhages are visible on the dorsal hard palate, mandibular mucosa, and ventral oropharynx.

friable spleen. We did not see any other lesions at necropsy.

What is the likely cause of the mandibular swelling and oral cavity ulcers? What tests could be performed to obtain a preliminary diagnosis? What differen- tial diagnoses should be considered? How would you treat and manage the frog and its colony?

\section{What's your diagnosis?}

